\title{
High CPAP vs. NIPPV in preterm neonates - A physiological cross-over study
}

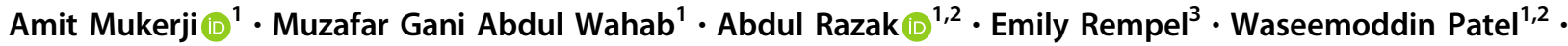 \\ Tapas Mondal ${ }^{1}$ - Jennifer Beck $\mathbf{B}^{4,5,6}$
}

Received: 10 March 2021 / Revised: 11 May 2021 / Accepted: 25 May 2021 / Published online: 5 June 2021

(c) The Author(s), under exclusive licence to Springer Nature America, Inc. 2021

\begin{abstract}
Objective To evaluate the physiological impact of high CPAP $\left(\geq 9 \mathrm{cmH}_{2} \mathrm{O}\right)$ vs. NIPPV at equivalent mean airway pressures. Study design In this cross-over study, preterm neonates on high CPAP or NIPPV were placed on the alternate mode. After $30 \mathrm{~min}$, left and right ventricular cardiac output and work of breathing indices were assessed, following which patients were placed back on the original mode and a similar procedure ensued.

Results Fifteen infants with mean (SD) postmenstrual age 32.7 (3.0) weeks, and weight 1569 (564) grams were included. No differences in LVO [320 (63) vs. 331 (86) $\mathrm{mL} / \mathrm{kg} / \mathrm{min}, P=0.46$ ] or RVO [420 (135) vs. 437 (141) $\mathrm{mL} / \mathrm{kg} / \mathrm{min}, P=0.19$ ] were noted during high CPAP vs. NIPPV, along with no differences in work of breathing indices.

Conclusion High CPAP pressures did not adversely impact cardiac output or work of breathing compared to NIPPV at equivalent mean airway pressure.
\end{abstract}

\section{Introduction}

Non-invasive respiratory support use has increased in the last decades in neonatal intensive care units to reduce the burden of lung injury caused by invasive mechanical

Supplementary information The online version contains supplementary material available at https://doi.org/10.1038/s41372021-01122-6.

Amit Mukerji

mukerji@mcmaster.ca

1 Department of Pediatrics, McMaster University, Hamilton, ON, Canada

2 King Abdullah bin Abdulaziz University Hospital, Princess Nourah Bint Abdulrahman University, Riyadh, Saudi Arabia

3 Department of Respiratory Therapy, Hamilton Health Sciences, Hamilton, ON, Canada

4 Keenan Research Centre for Biomedical Science of St. Michael's Hospital, Department of Critical Care, St. Michael's Hospital, Toronto, ON, Canada

5 Department of Pediatrics, University of Toronto, Toronto, ON, Canada

6 Member, Institute for Biomedical Engineering and Science Technology (iBEST) at Ryerson University and St-Michael's Hospital, Toronto, ON, Canada ventilation [1]. While there are a variety of non-invasive support modes, continuous positive airway pressure (CPAP) and non-invasive positive pressure ventilation (NIPPV) are typical forms [2]. Nasal CPAP delivers a constant distending pressure, whereas NIPPV delivers intermittent peak pressures above a constant distending pressure at set intervals to mimic tidal ventilation. Use of CPAP has traditionally been with positive end-expiratory pressure (PEEP) levels between 5 and $8 \mathrm{cmH}_{2} \mathrm{O}$ [3-5]. On the other hand, pressures and settings utilized with NIPPV use are highly variable [6,7], but typically result in mean airway pressures that are higher than traditional CPAP levels.

NIPPV is often used as a rescue form of non-invasive support when traditional CPAP is unable to support oxygenation and/or ventilation requirements. In recent metaanalyses of clinical trials that have compared CPAP and NIPPV [4, 5], the latter is suggested to be superior - with lower pooled estimates of failure. However, NIPPV use has limitations. The optimal pressure ranges that are effective and safe remain unknown resulting in tremendous practice variability [7]. Peak pressures are known to be diverted to the stomach [8], especially when high, often resulting in gastric distension and feeding intolerance. Furthermore, there is evidence that peak pressures are often asynchronous with patients' respiratory efforts, and even when 
synchronous, do not result in significant increases in measured tidal volume $[6,9]$. This has raised the possibility that reported clinical benefits of NIPPV might simply be a result of higher mean airway pressures, rather than the specific mechanism of gas flow delivery.

In recent years, use of high pressures during CPAP $(\geq 9$ $\mathrm{cmH}_{2} \mathrm{O}$ ) has emerged in the care of preterm neonates. In a recent survey of all Canadian tertiary level centres, 7 out of 28 sites reported use of CPAP pressures higher than traditional levels, suggesting emerging uptake of this practice [7]. However, there are limited data on the effectiveness and safety of high CPAP. Some preliminary physiological data have suggested safety $[10,11]$, while the only randomized clinical trial demonstrated that CPAP pressures between 7 and 9 compared to $4-6 \mathrm{cmH}_{2} \mathrm{O}$, when utilized post-extubation, resulted in fewer extubation failures in a cohort of extremely preterm neonates [12]. There are no published studies that have compared CPAP and NIPPV at equivalent mean airway pressures, despite the long-standing recognized need for such research [6]. The objective of this study was to compare the physiological impact of high CPAP vs. NIPPV at equivalent mean airway pressures.

\section{Methods}

\section{Study design}

This was a single-centre, prospective, cross-over, short-term study comparing high CPAP $\left(\geq 9 \mathrm{cmH}_{2} \mathrm{O}\right)$ vs. NIPPV and impact on physiological outcomes in preterm neonates.

\section{Study population}

Preterm infants without any congenital or chromosomal/ genetic abnormalities who were already being managed with either high CPAP (between 9 and $14 \mathrm{cmH}_{2} \mathrm{O}$ ) or NIPPV were screened for eligibility. At time of the study procedure, subjects had to meet following criteria: (a) >2 weeks' chronological age; (b) a postmenstrual age of at least 28 weeks (irrespective of gestational age); (c) current weight between 1000 and $2500 \mathrm{~g}$; (d) clinical stability with $\mathrm{FiO} 2<0.60$; and (e) informed and written parental consent. Clinical stability was further defined as no increased work of breathing on clinical assessment by medical team, no apneas or bradycardias requiring intervention, and no change in respiratory pressure settings within the previous $48 \mathrm{~h}$. In addition, any patients with an acute inter-current illness (including sepsis) or presence of hemodynamically significant ductus arteriosus (definition as shown in Supplementary File Table S1) were not included.
Finally, medical team approval was confirmed prior to any study procedure.

\section{Study procedure and data acquisition}

Patients on either high CPAP or NIPPV were first crossed-over to the alternate mode (e.g., high CPAP patient switched to NIPPV and vice versa) while ensuring the provision of equivalent mean airway pressure. For patients being switched from high CPAP to NIPPV, the conversion scheme as shown in Supplementary File (Table S2) was used to determine NIPPV settings. For patients on NIPPV, the calculated mean airway pressure was rounded to the nearest integer to choose the CPAP level. After a $30 \mathrm{~min}$ washout period, the cardiac output and electrical activity of diaphragm was recorded following which patient was crossed-over back to the original mode. Once back on the original mode, and another 30 min washout period, the cardiac output and electrical activity of diaphragm was ascertained once again for comparative analysis. Figure 1 depicts the study schema. The mean airway pressure as measured by the ventilator was documented every $10 \mathrm{~min}$ throughout the study procedure during both NIPPV and high CPAP. Both modes of support were provided using either a VN500 or Babylog 8000 (Drager, Lubeck, Germany) ventilators with appropriately sized masks or short bi-nasal prongs (Fisher and Paykel Healthcare Limited, Auckland, New Zealand). Neither the ventilator nor the interface type (mask/prong) was changed during the study for any individual participant.

\section{Assessment of cardiac output (Primary outcome)}

Cardiac output was evaluated for both left and right ventricles using 2-D echocardiograms, which was performed on any given subject for both high CPAP and NIPPV by either a neonatologist with formal training (MGAW, $N=13$ ) or a pediatric cardiologist (TM, $N=2)$. For the left ventricular output (LVO), the aortic root diameter was measured in duplicate, and the average value was used along with the subject's heart rate (HR), weight and velocity time-integral to calculate the $\mathrm{LVO}(\mathrm{mL} / \mathrm{kg} / \mathrm{min})$ using the following equation: $\quad \mathrm{LVO}=\frac{0.785 \times(A o R)^{2} \times V T i \times H R}{\text { Weight }}$. A similar set of measurements were performed to calculate RVO, using the pulmonary root diameter in place of aortic root diameter.

\section{Electrical activity of diaphragm (Secondary outcome)}

The minimum and peak electrical activity of the diaphragm (Edi), representing the work of breathing required to maintain functional residual capacity and generate tidal 
A

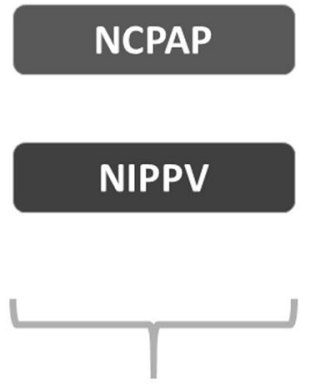

Original NRS settings*

*Patients on either mode will be eligible as long as mean Paw between 9-14 $\mathrm{cm} \mathrm{H}_{2} \mathrm{O}$
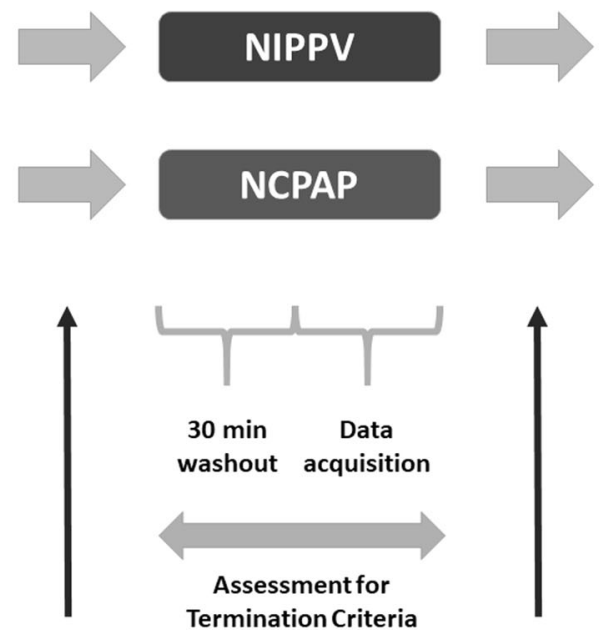

Study
commencement

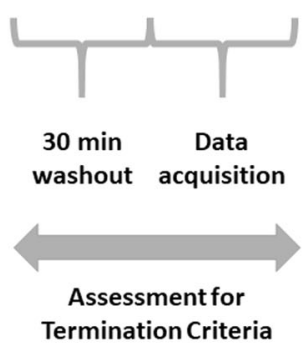

Termination Criteria

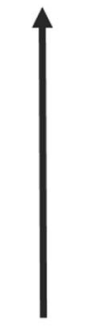

Crossover to original NRS mode \& settings

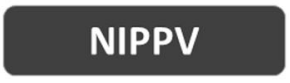

\section{NCPAP}

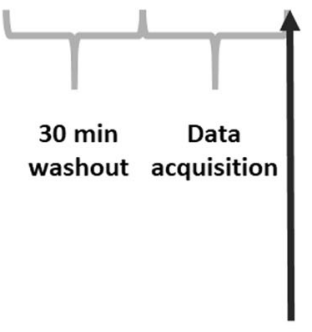

Study completion

Fig. 1 Study schema depicting the crossover between high CPAP and NIPPV at equivalent MAP. CPAP continuous positive airway pressure, MAP mean airway pressure, NIPPV non-invasive positive pressure ventilation.

ventilation, respectively, were measured for each subject using specialized Edi catheters (Maquet, Rastatt, Germany) [13]. The catheters were inserted in place of regular gastric tubes via the oropharynx and secured at an appropriate level as per manufacturer's recommendations. The Edi catheter was connected to a neurally adjusted ventilator assist module (Maquet, Rastatt, Germany) inserted into the ventilator, which then transmitted minimum and peak Edi signals to a data capturing software (Servo-Tracker v4.2, Maquet, Rastatt, Germany). The Edi waveform was analyzed for its peak at inspiration, minimum value at exhalation, and mean values in both inspiration and exhalation from a 5 min period from each mode per subject that provided the highest quality data, as previously described [14].

\section{Safety and termination criteria}

All subjects were monitored during the data acquisition period for pre-determined termination criteria, which included occurrence of any one of the following: (a) sustained increase/decrease in baseline HR $>20 \mathrm{bpm}$ above/ below baseline for $>10 \mathrm{~min}$; (b) Decrease in mean blood pressure $>10 \mathrm{mmHg}$ below baseline on two consecutive measurements being performed every $10 \mathrm{~min}$; (c) increase in $\mathrm{FiO}_{2}$ requirement $>10 \%$ above baseline for $>10 \mathrm{~min}$; and/ or (d) increase in work of breathing above baseline (as assessed by the Silverman score [15], and quantified by an increase in the score by $\geq 2$ ). The baseline levels of each of the above parameters were determined based on the mean value over $10 \mathrm{~min}$ on the original respiratory support mode immediately prior to initial cross-over. For baseline blood pressure, the average of two measurements taken $10 \mathrm{~min}$ apart was used.

\section{Sample size and data analysis}

Based on an alpha value of 0.05 , power set at $80 \%$ and previously reported standard deviations in cardiac output [16], a sample size of 15 subjects was selected to detect an a priori determined minimal clinically important difference of $50 \mathrm{~mL} / \mathrm{kg} / \mathrm{min}$ in cardiac output between high CPAP and NIPPV. Demographic data for all study subjects were summarized using descriptive statistics. All outcome measures were reported as means ( \pm standard deviation) and were compared using paired $t$-tests. Given the sample size, a post-hoc decision was made to also present standard error of means for cardiac output values. A $p$ value of $<0.05$ was considered to be significant. All final analyses were performed on GraphPad Prism v8 (GraphPad Software, San Diego, CA, USA).

\section{Results}

Fifteen patients underwent the study procedure and data acquisition between August 2017 and July 2020. The study procedure was performed in two additional patients who met inclusion criteria initially, but their data were not included in the study for the following reasons: in one patient, the Edi data was not captured during high CPAP due to a software error; in the second patient cardiac output from right ventricle was not estimable reliably due to a dysplastic pulmonary valve. For the final 15 subjects 
included in the study, the mean (SD) gestational age, chronological age, postmenstrual age and weight at time of study were 26.3 (0.8) weeks, 46 [20] days, 32.7 (3.0) weeks, and 1569 (564) grams, respectively. Table 1 depicts the baseline characteristics of individual patients, the settings on high CPAP and NIPPV, and the mean (SD) measured mean airway pressure on each mode for all patients.

Cardiac output [mean (SD/SEM)] was not different when evaluating either left [320 (63/16) vs. $331(86 / 22) \mathrm{mL} / \mathrm{kg} /$ $\min , P=0.46]$ or right ventricular output [420 (135/35) vs.
$437(141 / 36) \mathrm{mL} / \mathrm{kg} / \mathrm{min}, P=0.19]$ between high CPAP and NIPPV, respectively (Fig. 2). There were also no differences in the components of the individual parameters that contributed to the cardiac output calculations. The mean (SD) aortic velocity time-integral was 12.9 (2.6) vs. 13.4 $(2.9) \mathrm{cm}, P=0.12$ and mean (SD) HR was 165 [12] vs. 163 [12] bpm, $P=0.49$ during high CPAP and NIPPV during assessment of LVO, respectively. Similarly, during RVO measurements, the mean (SD) pulmonary velocity timeintegral was $13.7(3.4)$ vs. $14.1(3.2) \mathrm{cm}, P=0.19$ and the

Table 1 Individual-level demographics of patients included in the study, included original set and measured pressures on each mode of noninvasive support.

\begin{tabular}{|c|c|c|c|c|c|c|c|c|c|c|}
\hline Subject number & $\begin{array}{l}\text { GA } \\
\text { (weeks) }\end{array}$ & $\begin{array}{l}\text { BW } \\
\text { (grams) }\end{array}$ & Sex & DOL (days) & $\begin{array}{l}\text { PMA } \\
\text { (weeks) }\end{array}$ & $\begin{array}{l}\text { Current } \\
\text { weight } \\
\text { (grams) }\end{array}$ & $\begin{array}{l}\text { High } \\
\text { CPAP set } \\
\text { PEEP } \\
\left(\mathrm{cmH}_{2} \mathrm{O}\right)\end{array}$ & $\begin{array}{l}\text { Mean }(\mathrm{SD}) \\
\text { measured } \\
\text { MAP during } \\
\text { High CPAP } \\
\left(\mathrm{cmH}_{2} \mathrm{O}\right)\end{array}$ & $\begin{array}{l}\text { NIPPV Settings } \\
\text { (PIP/PEEP } \\
{\left[\mathrm{cmH}_{2} \mathrm{O}\right] \times} \\
\text { Rate }[\mathrm{bpm}])\end{array}$ & $\begin{array}{l}\text { Mean }(\mathrm{SD}) \\
\text { measured } \\
\text { MAP during } \\
\text { NIPPV } \\
\left(\mathrm{cmH}_{2} \mathrm{O}\right)\end{array}$ \\
\hline 1 & 25.42 & 800 & Female & 33 & 30 & 1330 & 11 & $11.0(0)$ & $17 / 8 \times 40$ & $11.0(0)$ \\
\hline 2 & 26 & 855 & Male & 34 & 30.28 & 1267 & 11 & $11.0(0)$ & $17 / 8 \times 40$ & $11.2(0.4)$ \\
\hline 3 & 25.14 & 740 & Male & 22 & 28.14 & 940 & 11 & $11.0(0)$ & $17 / 8 \times 40$ & $11.0(0)$ \\
\hline 4 & 27 & 870 & Male & 50 & 34.85 & 1785 & 9 & $9.0(0)$ & $13 / 7 \times 40$ & $9.1(0.4)$ \\
\hline 5 & 27.28 & 1100 & Female & 26 & 30.85 & 1280 & 11 & $11.1(0.4)$ & $16 / 8 \times 40$ & $10.8(0.4)$ \\
\hline 6 & 26.57 & 890 & Female & 49 & 33.42 & 1750 & 10 & $10.0(0)$ & $14 / 8 \times 40$ & $10.0(0)$ \\
\hline 7 & 27 & 984 & Female & 56 & 34.85 & 2143 & 10 & $10.0(0)$ & $14 / 8 \times 40$ & $10.0(0)$ \\
\hline 8 & 25.85 & 910 & Female & 23 & 28.85 & 1000 & 13 & $13.0(0)$ & $20 / 10 \times 40$ & $13.0(0)$ \\
\hline 9 & 26.14 & 893 & Male & 37 & 31.28 & 1460 & 9 & $9.0(0)$ & $13 / 7 \times 40$ & $9.0(0)$ \\
\hline 10 & 26.42 & 770 & Female & 67 & 35.85 & 2340 & 14 & $14.0(0)$ & $22 / 10 \times 40$ & $11.5(0.8)$ \\
\hline 11 & 26 & 675 & Female & 31 & 30.28 & 1120 & 12 & $14.2(1.2)$ & $20 / 8 \times 40$ & $12.4(1.5)$ \\
\hline 12 & 26 & 780 & Female & 31 & 30.28 & 1060 & 11 & $11.2(0.5)$ & $17 / 8 \times 40$ & $10.8(0.4)$ \\
\hline 13 & 25.28 & 872 & Male & 73 & 35.57 & 2310 & 9 & $8.8(0.2)$ & $13 / 7 \times 40$ & $8.9(0.2)$ \\
\hline 14 & 28.14 & 925 & Female & 41 & 33.85 & 1538 & 9 & $9.2(0.1)$ & $13 / 7 \times 40$ & $8.7(0.3)$ \\
\hline 15 & 25.85 & 440 & Female & 61 & 34.42 & 1170 & 9 & $9.1(0.1)$ & $13 / 7 \times 40$ & $9.4(0.3)$ \\
\hline
\end{tabular}

$B W$ birth weight, $C P A P$ continuous positive airway pressure, $D O L$ day of life, $G A$ gestational age, $M A P$ mean airway pressure, NIPPV noninvasive positive pressure ventilation, $P E E P$ positive end-expiratory pressure, $P I P$ peak inspiratory pressure, $P M A$ postmenstrual age, $S D$ standard deviation.

A

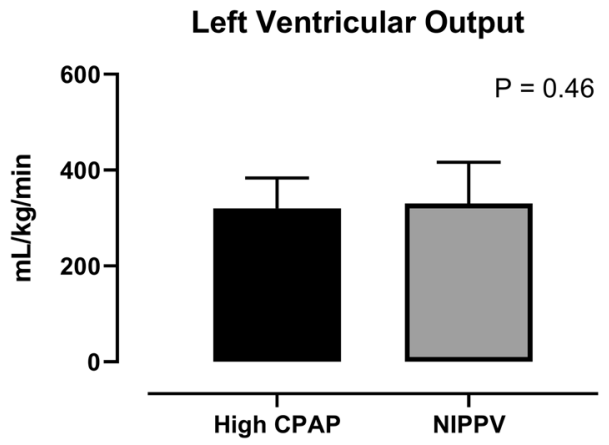

Fig. 2 Cardiac Output on High CPAP vs. NIPPV. A LVO estimates were 320 [63] vs. 331 [86] mL/kg/min, $P=0.46$ during high CPAP and NIPPV, respectively. B RVO values were 420 (135) vs. 437 (141) mL/kg/
B

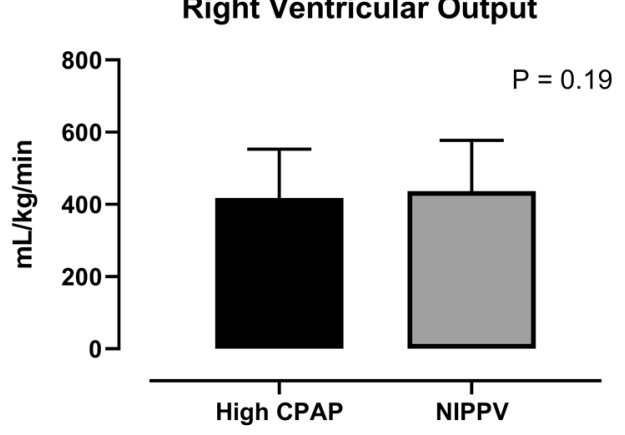

min, $P=0.19$ with high CPAP and NIPPV, respectively. CPAP continuous positive airway pressure, LVO left ventricular output, NIPPV nasal intermittent positive pressure ventilation, RVO right ventricular output. 
A

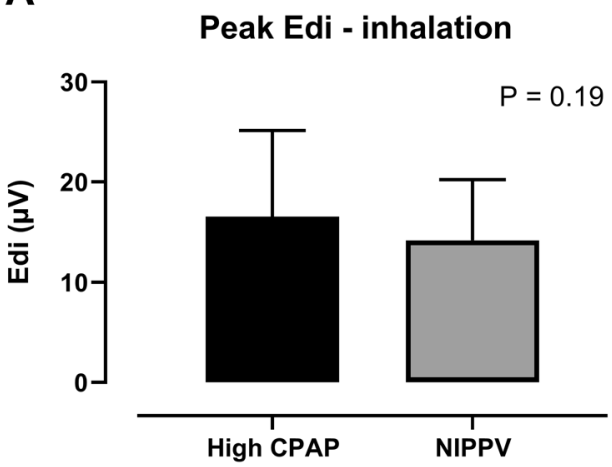

Fig. 3 Peak and minimum Edi on High CPAP vs. NIPPV. A Peak Edi values assessed during inhalation were 16.6 (8.6) vs. 14.2 (6.0) $\mu \mathrm{V}, P=0.19$ during high CPAP and NIPPV, respectively. B Minimum Edi values assessed in expiratory phase were 6.2 (3.0) vs. 5.4

mean (SD) HR was 162 [11] vs. 162 [13] bpm, $P=0.84$ with high CPAP and NIPPV, respectively. The Edi data did not demonstrate any statistically significant differences between high CPAP and NIPPV (Fig. 3). In addition, no study subject met pre-determined study termination criteria based on assessment of clinical physiological parameters. Finally, a post-hoc sensitivity analysis was conducted after excluding participant numbers 10 and 11 who had discrepant measured mean airway pressures during high CPAP vs. NIPPV (Table 1), results of which showed no difference in cardiac output or Edi values (Supplementary File Table S3).

\section{Discussion}

\section{Summary of results}

In this cohort of 15 preterm neonates who were already on either high CPAP or NIPPV, there was no impact on either left or right ventricular output when crossed-over to the alternate mode as measured by 2-D echocardiography. There was no significant change in work of breathing indices as measured using electrical activity of the diaphragm. All study subjects tolerated the cross-over for the duration of the study period without any changes in clinical physiological parameters.

\section{Significance of results}

With ever-increasing use of non-invasive respiratory support modes, it is important to generate evidence to ultimately inform clinical guidelines for best practice. High CPAP use is emerging as an attractive alternative to NIPPV despite limited data on indications, effectiveness, and safety. It may indeed confer advantage for individual patients by virtue of
B

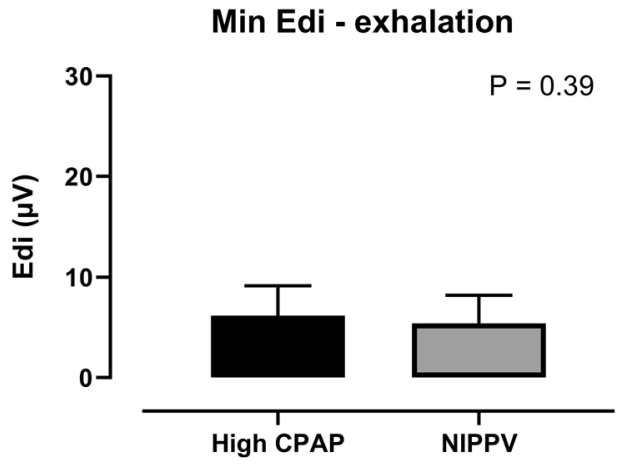

(2.8) $\mu \mathrm{V}, P=0.39$ during high CPAP and NIPPV, respectively. CPAP continuous positive airway pressure, Edi electrical activity of diaphragm, NIPPV nasal intermittent positive pressure ventilation.

maintaining a constant high distending pressure without the periodic peak pressure transmissions and associated limitations during NIPPV [6]. However, till date there are limited data comparing high CPAP and NIPPV at equivalent mean airway pressures, with no studies to our knowledge that have evaluated the physiological impact.

In the first study of its kind, the impact of high CPAP vs. NIPPV at equivalent mean airway pressure on cardiac output and work of breathing was evaluated. Consistent with our hypothesis, there were no differences in either left or right ventricular output when comparing the two modes. Interestingly, the RVO measurements were noted to be higher than LVO although these were not intended to be compared against each other. We speculate this difference may result from slightly higher measurement estimates of the pulmonary root, which was due to measurements taken at a slightly higher level compared to the aortic root. In relation to work of breathing indices, while no differences were noted, there was a trend toward lower peak and minimum Edi signals with NIPPV, a finding that deserves further exploration in appropriately powered studies.

\section{Comparison with previous literature}

Hsu et al. compared varying levels of CPAP on patients who were originally in room air, reporting a decline in cardiac output when comparing no CPAP to CPAP at $10 \mathrm{cmH}_{2} \mathrm{O}$ [17]. On the other hand, Mukerji et al. reported no changes in cardiac output when comparing varying levels of CPAP ranging from 5 to $13 \mathrm{cmH}_{2} \mathrm{O}$ in a cohort of stable infants [11]. In that study, LVO and Edi were assessed and interestingly, the LVO $(295 \pm 75 \mathrm{~mL} / \mathrm{kg} / \mathrm{min})$ and Edi values (peak Edi $15.4 \pm 10.6 \mu \mathrm{V}$ and min Edi $4.2 \pm$ $4.6 \mu \mathrm{V}$ ) at $\mathrm{CPAP} 5 \mathrm{cmH}_{2} \mathrm{O}$ were similar to those on high CPAP and NIPPV in the present study. However, both these studies reported on patients who required less respiratory 
support than the present cohort, precluding formal statistical comparisons. Gupta et al. reported preliminary results of the clinical impact of CPAP vs. NIPPV at equivalent pressures, demonstrating no differences in the primary outcome of bradycardias [10]. However, not all patients in this cohort were on high CPAP pressures $>8 \mathrm{cmH}_{2} \mathrm{O}$. Binmanee et al. reported short-term clinical outcomes with use of high pressures on non-invasive support (which included high CPAP) and reported success with prevention of invasive mechanical ventilation, but was limited by lack of control group [18]. The only randomized clinical trial compared CPAP $4-6 \mathrm{cmH}_{2} \mathrm{O}$ vs. $7-9 \mathrm{cmH}_{2} \mathrm{O}$ postextubation and reported lower rates of extubation failure with the higher pressure group [12]. The increasing use of high CPAP despite the limited available scientific substantiates the clear need for further research. Ours is the first study that compares physiological outcomes when comparing high CPAP with NIPPV at equivalent mean airway pressures.

\section{Weaknesses and strengths}

Some key limitations of this study should be recognized. The variability in cardiac output was higher than anticipated that were used as a basis for sample size calculations. As such, the possibility of a type II error exists. However, as the absolute values of both left and right ventricular output were very similar, it is uncertain whether a larger sample size would have changed the outcome. Furthermore, it must be acknowledged that echocardiograms as a tool to measure cardiac output may have limited reliability due to technical challenges, although it remains the gold standard in neonates [19]. Also, while we focused on LVO and RVO, we did not include assessment of preload as a marker of hemodynamic compromise in this study. Our results for the work of breathing indices were not powered due to it being an exploratory outcome. As well, despite the need for high non-invasive pressures, included patients were generally stable which limits generalizability to patients who are acutely unwell. In addition, we did not pursue any clinical outcomes as they were deemed outside the scope of this targeted study. Finally, the measured pressures during both high CPAP and NIPPV showed variability in some patients, highlighting the importance of continuous clinical vigilance in optimizing the interface securement to deliver the set measures as best as possible.

The major strength is this being the first study to evaluate the physiological impact of high CPAP and NIPPV at equivalent pressures on cardiac output and work of breathing. The cross-over design provided consistency for purposes of comparison. Finally, clinical parameters were assessed for safety and termination based on a priori determined cut-offs during the cross-over, although no subjects met these criteria.

\section{Conclusions and future directions}

In this study, cardiac output was not adversely impacted by use of high CPAP pressures at equivalent mean airway pressure as NIPPV. However, studies with larger sample sizes are needed to be definitive. Work of breathing indices also require further evaluation, as is the need for prospective clinical trials toward determining the optimal use of high CPAP and impact on both short and long-term clinical outcomes.

Acknowledgements Authors would like to acknowledge the following individuals/groups: (A) Norm Comtois in the lab of JB for performing Edi data analyses; (B) respiratory therapists at McMaster Children's Hospital's neonatal intensive care unit for their support during conduct of the study; and (C) Aravanan Chakkarapani, MD for writing the first draft of the study protocol.

Author contributions Each listed author has met criteria for authorship requirements. AM devised the study concept. AR and AM developed the study protocol. AR, WP, ER, AM, TM and MGAW conducted the study procedure. AM and JB conducted the analyses. AM wrote the paper. All authors reviewed and approved the study paper.

Funding Regional Medical Associates Research Scholarship Grant, Hamilton Health Sciences. Dr AM is supported by a Research Early Career Award from Hamilton Health Sciences Foundation (2019-21).

\section{Compliance with ethical standards}

Conflict of interest AM, MGAW, AR, ER, WP and TM have no potential competing interests to disclose. JB has made inventions related to neural control of mechanical ventilation that are patented. The patents are assigned to the academic institution(s) where inventions were made. The license for these patents belongs to Maquet Critical Care. Future commercial uses of this technology may provide financial benefit to Dr JB through royalties. Dr JB owns $50 \%$ of Neurovent Research Inc (NVR). NVR is a research and development company that builds the equipment and catheters for research studies. NVR has a consulting agreement with Maquet Critical Care.

Ethical approval The study protocol was approved by the local research ethics board (Hamilton integrated ethics board approval number: 2283). Written informed consent was obtained from parent(s) of each study participant.

Publisher's note Springer Nature remains neutral with regard to jurisdictional claims in published maps and institutional affiliations.

\section{References}

1. Sammour I, Karnati S. Non-invasive respiratory support of the premature neonate: from physics to bench to practice. Front Pediatr. 2020;8:214.

2. Mahmoud RA, Roehr CC, Schmalisch G. Current methods of non-invasive ventilatory support for neonates. Paediatr Respir Rev. 2011;12:196-205.

3. Diblasi RM. Nasal continuous positive airway pressure (CPAP) for the respiratory care of the newborn infant. Respir Care. 2009;54:1209-35. 
4. Lemyre B, Laughon M, Bose C, Davis PG. Early nasal intermittent positive pressure ventilation (NIPPV) versus early nasal continuous positive airway pressure (NCPAP) for preterm infants. Cochrane Database Syst Rev. 2016;12:Cd005384.

5. Lemyre B, Davis PG, De Paoli AG, Kirpalani H. Nasal intermittent positive pressure ventilation (NIPPV) versus nasal continuous positive airway pressure (NCPAP) for preterm neonates after extubation. Cochrane Database Syst Rev. 2017;2:Cd003212.

6. Owen LS, Manley BJ. Nasal intermittent positive pressure ventilation in preterm infants: Equipment, evidence, and synchronization. Semin Fetal Neonatal Med. 2016;21:146-53.

7. Mukerji A, Shah PS, Shivananda S, Yee W, Read B, Minski J, et al. Survey of noninvasive respiratory support practices in Canadian neonatal intensive care units. Acta Paediatr. 2017;106: 387-93.

8. Hadj-Ahmed MA, Samson N, Nadeau C, Boudaa N, Praud JP. Laryngeal muscle activity during nasal high-frequency oscillatory ventilation in nonsedated newborn lambs. Neonatology. 2015;107:199-205.

9. Owen LS, Morley CJ, Dawson JA, Davis PG. Effects of nonsynchronised nasal intermittent positive pressure ventilation on spontaneous breathing in preterm infants. Arch Dis Child Fetal Neonatal Ed. 2011;96:F422-8.

10. Gupta AK, Szynal A, Hallinan S, Keszler M. CPAP vs. Unsynchronized NIPPV at Equal Mean Airway Pressure (MAP). Pediatr Academic Societies. 2019;2851:442.

11. Mukerji A, Wahab MGA, Mitra S, Mondal T, Paterson D, Beck $J$, et al. High continuous positive airway pressure in neonates: a physiological study. Pediatr Pulmonol. 2019;54: 1039-44.

12. Buzzella B, Claure N, D’Ugard C, Bancalari E. A randomized controlled trial of two nasal continuous positive airway pressure levels after extubation in preterm infants. J Pediatr. 2014;164: 46-51.

13. Stein H, Firestone K. Application of neurally adjusted ventilatory assist in neonates. Semin Fetal Neonatal Med. 2014;19:60-9.

14. Beck J, Reilly M, Grasselli G, Qui H, Slutsky AS, Dunn MS, et al. Characterization of neural breathing pattern in spontaneously breathing preterm infants. Pediatr Res. 2011;70:607-13.

15. Hedstrom AB, Gove NE, Mayock DE, Batra M. Performance of the Silverman Andersen Respiratory Severity Score in predicting $\mathrm{PCO}(2)$ and respiratory support in newborns: a prospective cohort study. J Perinatol. 2018;38:505-11.

16. Abdel-Hady H, Matter M, Hammad A, El-Refaay A, Aly H. Hemodynamic changes during weaning from nasal continuous positive airway pressure. Pediatrics. 2008;122:e1086-90.

17. Hsu HS, Chen W, Wang NK. Effect of continuous positive airway pressure on cardiac output in neonates. Zhonghua Min Guo Xiao Er Ke Yi Xue Hui Za Zhi. 1996;37:353-6.

18. Binmanee A, El Helou S, Shivananda S, Fusch C, Mukerji A. Use of high noninvasive respiratory support pressures in preterm neonates: a single-center experience. J Matern Fetal Neonatal Med. 2017;30:2838-43.

19. Weisz DE, Jain A, McNamara PJ, EL-Khuffash A. Non-invasive cardiac output monitoring in neonates using bioreactance: a comparison with echocardiography. Neonatology. 2012;102:61-7. 